\title{
Agôn
}

Revue des arts de la scène

5 | 2012

L'entrée en scène

\section{Entrer en reflet : l'entrée en scène dans des dispositifs optiques}

Entretien réalisé par Anne Pellois

Joris Mathieu, Philippe Chareyron et Anne Pellois

\section{OpenEdition}

Journals

Édition électronique

URL : http://journals.openedition.org/agon/2412

DOI : 10.4000/agon.2412

ISSN : 1961-8581

Éditeur

Association Agôn

Référence électronique

Joris Mathieu, Philippe Chareyron et Anne Pellois, « Entrer en reflet : l'entrée en scène dans des dispositifs optiques », Agôn [En ligne], 5 | 2012, mis en ligne le 25 janvier 2013, consulté le 14 septembre 2020. URL : http://journals.openedition.org/agon/2412

Ce document a été généré automatiquement le 14 septembre 2020.

Association Agôn et les auteurs des articles 


\title{
Entrer en reflet : l'entrée en scène dans des dispositifs optiques
}

\author{
Entretien réalisé par Anne Pellois
}

Joris Mathieu, Philippe Chareyron et Anne Pellois

\section{NOTE DE L'ÉDITEUR}

Entretien réalisé le 13 juin 2012

Joris Mathieu est metteur en scène et fondateur de la compagnie Haut et Court depuis 1998. Philippe Chareyron est comédien au sein de la compagnie. À la recherche d'un théâtre « extra ordinaire », les spectacles de Haut et Court travaillent l'illusion optique, par la présence trouble des comédiens et la mise en place de dispositifs comme le théâtre optique ou la déambulation, qui perturbent les modalités de l'entrée en scène et sa perception, questionnées ici du double point de vue du metteur en scène et du comédien.

\section{Entrer dans un dispositif optique}

Anne pelLoIs : Vous venez de donner ensemble, Joris Mathieu en tant que metteur en scène et Philippe Chareyron en tant que comédien principal, Urbik/Orbik, spectacle autour de l'univers de Philippe K.Dick, dans un dispositif de théâtre optique. Pourriez-vous commencer par une description de ce dispositif, pour voir en quoi la question de l'entrée en scène se pose de manière singulière dans cet espace?

Joris MATHIEU : Il y a trois états de l'acteur en scène : l'acteur au plateau qui joue en live ; L'acteur en différé via des images filmées qui sont diffusées; l'acteur en live, et en miroir, réfléchi sur la scène. Dans ce dernier cas l'acteur joue dans une fosse. Cette fosse se reflète dans une paroi réfléchissante et l'œil du spectateur voit les comédiens en scène. L'acteur joue donc sous le plateau, au sol, allongé, dans d'autres rapports à l'espace. Il faut par exemple être allongé pour que la verticalité soit produite. Il y a donc ces trois états de jeu, et il y a un travail à faire pour que malgré les différentes 
natures de corps, la ligne directrice soit toujours la même. Cela nous amène à tirer le jeu de l'acteur, quand il est en scène de manière classique au plateau, vers ce à quoi ressemblent les autres états. Parce qu'on ne peut pas demander à la vidéo de ressembler à l'acteur charnel et à un acteur allongé de se comporter et de se mouvoir de la même manière que s'il était debout sur scène. On est donc obligé de modifier la partition de jeu au plateau pour que l'unité existe. D'où ce rapport éthéré de l'acteur à la scène, qui m'intéressait parce que cela posait la question de l'état de jeu et du climat, et plus précisément la question de la force de l'acteur pour fabriquer ce climat. Beaucoup croient que c'est la musique ou le dispositif qui le fabriquent. Certes ils y participent, mais c'est surtout fondamentalement la façon dont les comédiens se meuvent, l'état dans lequel ils arrivent sur scène, qui crée cela. Quand on a commencé à travailler avec deux jeunes comédiens qui sortaient de la Comédie de Saint Étienne, Marc et Odile ${ }^{1}$, on s'est très vite rendu compte qu'ils ne trouvaient pas leurs marques dans le dispositif et entraient en résistance, notamment Marc qui voulait revendiquer son statut d'acteur, ce pourquoi il faisait du théâtre : porter la parole, porter la voix, mettre du mouvement, de l'action. Et dès qu'il rentrait en résistance, tout s'effondrait. L'acteur doit se familiariser avec ce type de rapport à la scène. En fait, l'entrée en scène n'intervient pas tant au moment où la représentation commence, qu'une ou deux heures avant, dans la préparation à ce travail spécifique, dans l'acceptation de ce rapport particulier que l'on va avoir au public. Ensuite, il y a des questions plus classiques comme celle du timing par exemple, qui ne diffèrent pas tellement des autres spectacles.

Urbik/Orbik, Comédie de Caen

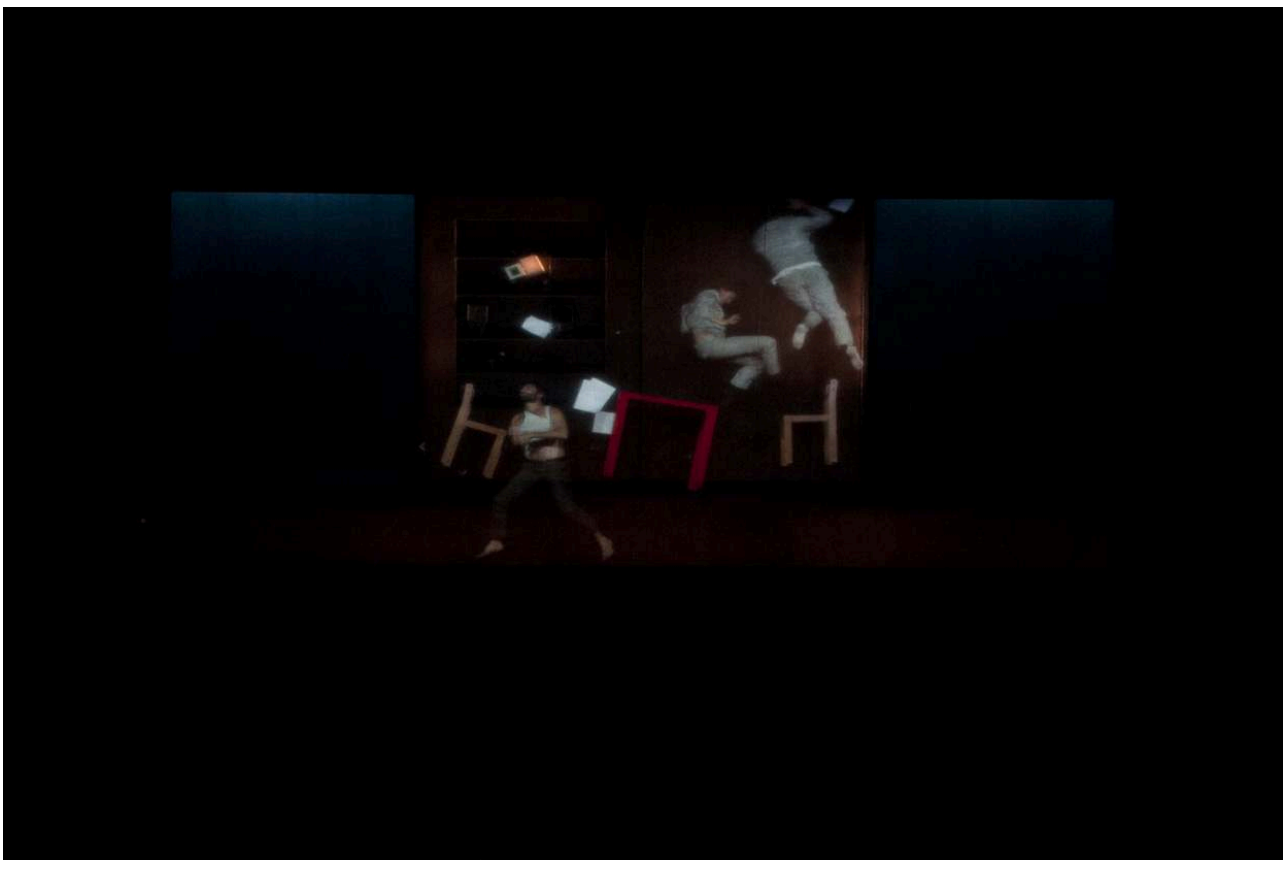

Nicolas Boudier

AP: Est-ce que vous vous posez la question de l'entrée en scène de manière centrale lorsque vous travaillez?

$\mathrm{JM}$ : Généralement on prend la question de l'entrée en scène à rebours. Souvent dans les derniers spectacles, et c'est le cas dans Urbik/Orbik, les scènes s'ouvrent avec les 
comédiens déjà en place. Les entrées sont plus des apparitions en lumière ou des apparitions dans le dispositif du théâtre optique. Finalement, quand l'acteur a à entrer en scène, il intervient dans un tableau qui est déjà installé. Dans ce cas-là, l'entrée en scène est conditionnée par le climat posé au préalable. Tout le travail est de se fondre dans ce climat. Le spectacle précédent, Des Anges mineurs ${ }^{2}$, avait une mise en scène somme toute assez classique, avec des entrées à cour et à jardin, mais on manquait de profondeur. Dans Urbik, il y a beaucoup moins de sortie, et les entrées se font de manière beaucoup plus virtuelle.

Philippe ChAREYRon: Plus que l'entrée en scène, c'est la question de la mise en jeu dans ce dispositif qui est importante: dans quel climat on s'insère, comment on préexiste en tant qu'acteur à l'apparition ou à l'entrée en scène, comment on joue de loin, de la coulisse ou du noir, comment enfin on est en jeu quand on n'est pas à vue.

AP : Si l'on part du principe que vous pensez l'entrée en scène comme une apparition, et non comme un franchissement, faites-vous une différence, en tant qu'acteur, entre franchir le seuil de la coulisse et apparaître en lumière?

PC : Non, pas vraiment parce qu'on essaye d'être dans un fil de jeu, de ne pas sortir de ce qu'on doit jouer, d'être tout le temps à l'intérieur pour dérouler la pelote. Le parcours continue aussi bien à l'extérieur du plateau que dans un noir du plateau. C'est dans ce sens-là qu'on influe sur le jeu et sur la façon d'apparaître.

$\mathrm{JM}$ : Même dans les temps de hors-jeu, c'est-à-dire dans le temps où les comédiens ne sont pas au plateau, on se débrouille toujours pour leur trouver des choses à faire, en terme de machinerie, de mise en place. Certes, l'acteur sort du jeu, mais c'est aussi une façon de maintenir l'état actif en permanence. Même si on essaye de ne pas tomber dans l'excès inverse - qui perturberait trop l'attention d'un comédien trop sollicité - l'acteur est toujours là, toujours actif, et participe à animer la machine qui crée l'histoire et l'illusion pour le spectateur. C'est une autre façon d'être encore en scène. Dans Urbik, il y a des scènes où les acteurs ne sont pas au plateau. Depuis les coulisses, on voit un plateau vide devant lequel est rassemblé un parterre de spectateurs qui eux voient des choses. Cela pose beaucoup de questions sur la place de l'acteur et sur la manière de s'amuser avec cette adresse différée, surtout quand les acteurs n'entrent même pas en scène. Ils sont dans des fosses, ils apparaissent, ils jouent mais ils n'entrent pas. Il n'y a pas de franchissement. Ils sont même souvent contraints dans le corps, dans des positions induites par le dispositif. Et malgré tout, la rencontre se fait avec le spectateur. Ce qui est moteur pour nous dans le rapport à l'entrée en scène, c'est que l'acteur ne rentre jamais pour être vu, mais pour être aperçu, deviné, inclus dans autre chose.

PC : Il y a ce côté magique de la machine qui va nous faire apparaitre, par le biais de la vidéo, des techniciens en régie. On se sent comme un rouage parmi tant d'autres, et on se dit qu'on a plutôt intérêt à maintenir le cap puisqu'un grain de sable peut dérégler l'ensemble.

AP : Est-ce différent d'entrer sur le plateau en étant physiquement sur le plateau, et d'entrer en jeu dans la fosse?

PC : C'est presque pareil, sauf en terme de postures. On met plus de temps à régler les choses dans la fosse, mais l'intensité du jeu doit être la même. C'est une autre excitation de jouer dans la fosse parce qu'on sait qu'on va créer une illusion en direct. 
Par exemple, dans la séquence où l'on flotte dans l'air, je suis dans un mouvement tout petit, tout idiot, et les gens ont vraiment l'impression que je flotte.

\section{AP : La première entrée est-elle la plus importante ?}

PC : En ce qui me concerne, c'est clair : le moment crucial c'est le début. Une fois que c'est parti, c'est parti. Le seul moment un peu tangent, il est là. Une fois que les choses sont lancées, cela ne s'arrête plus. C'est un vrai plaisir de jeu d'avoir ses marques, de maîtriser le déroulé. Le moment crucial, c'est l'ouverture.

JM : C'est à nuancer à mon avis, selon les rôles. Dans Des Anges mineurs, les trois comédiens au plateau étaient en permanence mobilisés et sollicités. Sur Urbik/Orbik, Philippe est en scène en permanence et les autres font des entrées. On entre par les premiers mots, ce sont les premiers rails, et cela conditionne fondamentalement la représentation. C'est valable pour tous les comédiens. Pour les rôles plus secondaires qui viennent alimenter la narration, il se joue quelque chose de déterminant à chaque entrée. Certes, la nécessité d'entrer est écrite dans la partition du spectacle, mais il reste des espaces de liberté à l'intérieur de cette partition. Il n'y a pas tant de repères auxquels s'accrocher. Je fais plus confiance à l'intuition et à l'engagement de l'acteur quand la représentation commence qu'à sa fidélité scrupuleuse à reproduire des marques et des repères.

\section{Des Anges mineurs. Vénissieux}

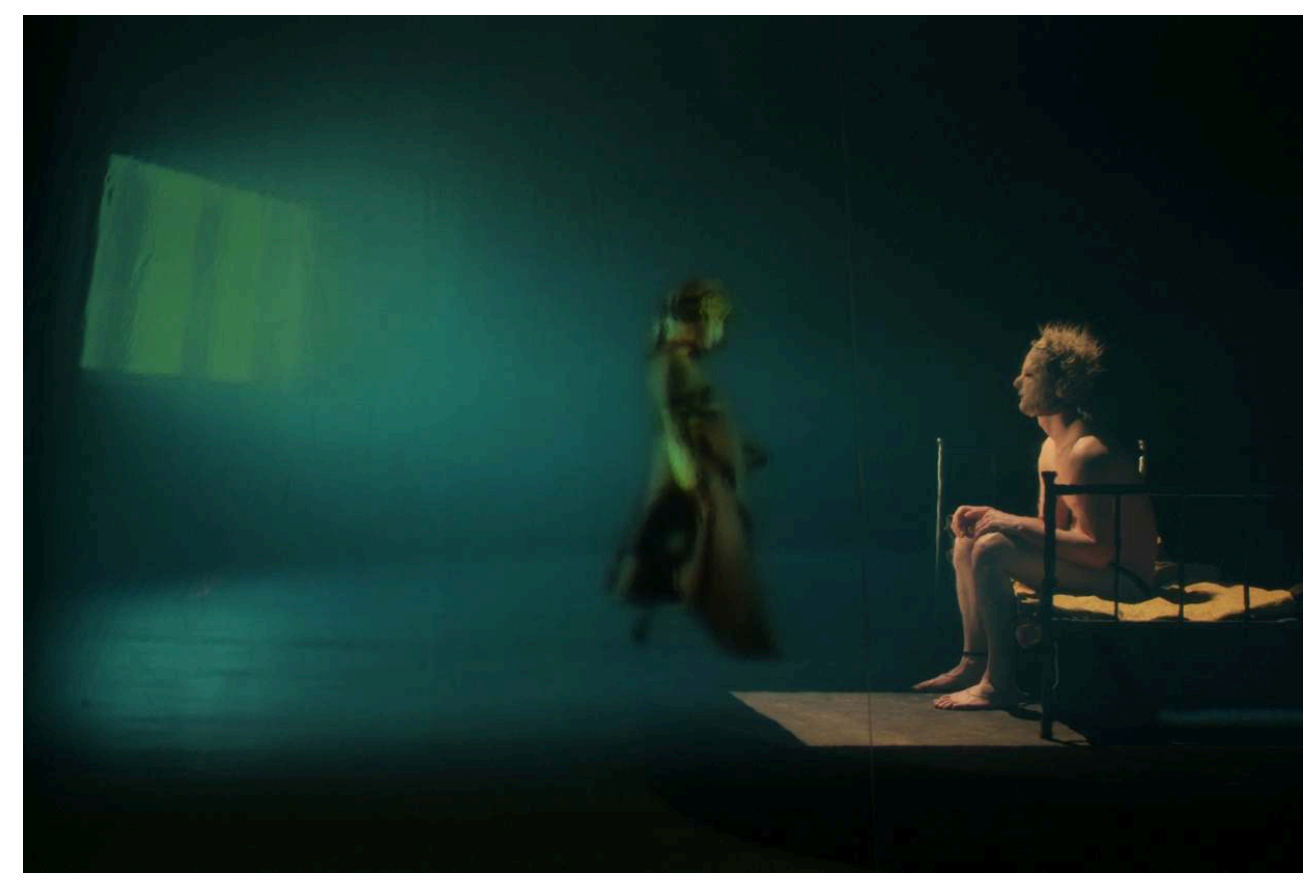

Nicolas Boudier

AP : De l'extérieur, en tant que spectateur, on a l'impression que c'est quand même très contraignant, que ce dispositif conditionne de manière plus serrée qu'un simple plan de feu les entrées et les sorties des comédiens.

$\mathrm{JM}$ : Bien sûr, le cadre est très technique, mais pas plus contraignant qu'un plan de feu. 
PC : La magie du spectacle vivant, c'est que c'est toujours le comédien qui reste au centre. Les techniciens suivent. Nous à l'intérieur, on mène le jeu. On a la liberté de se tromper sans que ça mette forcément tout le spectacle en danger.

$\mathrm{JM}$ : La difficulté c'est le rapport au public qui n'est pas immédiat. Ce que les acteurs voient du public, ce sont des reflets. Autant les spectateurs doivent forcer leur attention pour croire qu'il y a quelqu'un sur scène, autant les acteurs doivent se convaincre que quelqu'un les regarde. L'acteur ne peut pas se reposer sur un rapport d'énergie ou de connivence avec le spectateur. Il est vraiment dans la recherche de sa place à l'intérieur de l'histoire. La perception des autres il ne peut pas la ressentir.

AP : Ce qui fait la beauté de ces spectacles-là, c'est qu'on ne bascule pas complètement du côté de l'image, et qu'il reste des présences dont on ne sait pas toujours d'ailleurs si elles sont réelles ou pas. Est-ce que vous pourriez dire que vous « entrez en image » ou bien estce que vous avez toujours la sensation d'entrer en scène?

PC : J'ai toujours l'impression d'être en scène, jamais d'être une image. On joue et on croit à ce que l'on joue. Faire sentir qu'il y a encore cette chair de l'acteur, c'est très important et cela nécessite d'être en scène, de réfléchir à la manière d'être en scène en traversant ces filtres et ces miroirs. Au début de la création les choses n'arrivaient pas complètement à prendre corps.

JM : Je ne vois pas bien la différence entre « être en scène » et « être en image ». Dans d'autres spectacles, on n'utilise pas du tout l'image numérique. Pour moi, l'espace du plateau a toujours été un espace pictural, architecturé, construit, ce qui fait que l'acteur est toujours dans ce rapport-là, à la fois dans un univers plastique, esthétique, et dans une position d'actant, d'orateur, de moteur de l'action ou de l'inaction. Si l'image est cet espace dans lequel évoluent des êtres et des personnages, si notre perception de spectateur fait évoluer celle-ci comme une narration, alors j'assume volontiers l'idée de l'acteur dans l'image, sans y voir nécessairement l'acteur dissimulé à l'intérieur d'une image, mais plutôt l'acteur construisant des images et une histoire pour le spectateur. Pour moi, l'espace de la scène et celui de l'image, ce sont les mêmes.

PC: On est en scène, mais on n'a de cesse de construire des images, faites pour toucher. De temps en temps, on se sort de la scène pour regarder si c'est beau ce qu'on a fait, et pour avoir conscience de ce que ça fait.

$\mathrm{JM}$ : On est aussi sur de l'image en 3D et pas 2D. On peut se dire que l'espace de la scène et l'espace de l'image sont les mêmes, puisqu'on n'est pas dans une image à deux plans, écrasés. On est toujours dans de l'animé. Même les images brutes qu'on utilise sont construites pour l'espace théâtral. Ce sont rarement des images filmiques en tant que telles, des images qui cherchent à avoir une esthétique cinématographique, mais des images construites pour apparaître sur la scène et pour permettre au comédien de s'y insérer. Et ça pour moi c'est fondamental.

\section{Faire entrer le spectateur : mise en mouvement, aspiration, hypnose.}


AP : Est-ce qu'on peut envisager dans un dispositif où le spectateur déambule, comme Le $B a r d 0^{3}$, par exemple, la question de l'entrée dans l'espace de jeu du spectateur?

PC : On se pose toujours la question de savoir dans quelle atmosphère on plonge les gens et dans quel état ils arrivent. On instaure souvent un sas de compression ou décompression, qui permet de mettre les spectateurs dans un certain état. Dans Urbik, c'est la fonction du long monologue liminaire de ce personnage fantomatique qui accueille les spectateurs, d'abord dans la lumière de la salle avec une voix qui prend petit à petit le dessus pour plonger les gens dans une sorte d'hypnose. Dans Le Bardo, on discute avant que le spectateur n'entre dans le labyrinthe, on lui raconte un peu ce qui va se passer et comment cela va se passer. On essaye de faire en sorte que ce ne soit jamais abrupt. On convoque les gens dans un univers assez fort, tout le temps, et on ne se donne pas le droit de les malmener en les plongeant directement dans un bain d'huile.

JM : L'idée est de plonger et d'accompagner les spectateurs dans une plongée, dans une immersion. Dans Le Bardo, c'est un peu différent : les gens se préparent à rentrer seuls dans une pièce obscure après un temps d'attente pendant lequel on essaye de construire quelque chose. Toute la dramaturgie du Bardo, c'est, par la traversée des chambres successives, de mettre le spectateur en mouvement sans contrainte, de l'orienter plutôt que de flécher son parcours. Dans ce spectacle, on se pose plus la question de l'entrée en scène du spectateur que de celle du comédien. Comment va-til entrer dans la chambre ? Comment va-t-il sentir que c'est le moment de quitter cet endroit? Comment lui donner envie d'aller plus loin, malgré les peurs qui peuvent apparaître?

AP : Le dispositif d'Urbik/Orbik fait l'effet d'un trou noir. Le spectateur est comme aspiré par le cadre de scène, hypnotisé, notamment à cause des difficultés de perception induites par la pénombre. Est-ce une manière de faire entrer le spectateur dans le spectacle?

PC : On adore que les gens disent qu'ils ont fait une sorte de rêve. Mais si on les plongeait brutalement dedans, ce serait plutôt une sorte de cauchemar. Sans manipuler les gens, il faut trouver le moyen de les faire changer d'état, de les hypnotiser, de les mettre en disponibilité pour qu'ils acceptent de nous accompagner. Se retrouver dans le labyrinthe du Bardo sans savoir ce qu'il va se passer, si on va être dans le noir ou non, c'est un effort conséquent. À nous de faire en sorte qu'ils acceptent nos petites règles.

JM : J'aime bien cette idée, que j'ai formulée quand on travaillait sur Le Bardo, que le théâtre n'existe pas vraiment, ou plutôt qu'il n'existe que parce qu'on veut bien le voir. Et cela ne fonctionne que par la volonté et la participation active du spectateur. À ce titre, on s'agite, ou bouge, on tire des ficelles, on fait fonctionner des poulies, on entre en scène, on met de la musique. On crée le contexte favorable pour que le théâtre puisse exister, pour que la vision existe. Mais finalement, le travail, c'est le spectateur qui le fait. Et c'est vrai pour Les Anges mineurs, pour Urbik/Orbik, encore plus dans le Bardo. On donne toujours des espaces qui sont très cadrés, dans lesquels les images sont très construites. Tout donne l'impression d'être très cadré et très propre. Et la question du hors-champ, du hors-cadre, la question de la provenance, se déplace, c'est-à-dire que c'est plutôt d'où je viens moi et où je vais moi qui regarde. Et dans quoi je me plonge. Et le hors-champ c'est tout ce avec quoi le spectateur arrive. 
AP : Tu dis hors-champ, tu ne dis pas hors-scène !

JM : Pour moi, c'est le champ de la perception, de la vision. Je pense vraiment que pour le spectateur, il est plus question de hors-champ, puisque la scène est tout le temps maquillée. L'espace scénique n'apparaît jamais en tant que tel.

AP : Philippe, tu as évoqué tout à l'heure la nécessité de faire passer le spectateur dans un sas de compression. Qu'est-ce que tu entends par là ?

PC : Cette idée de sas de décompression m'est venue un jour, je m'en souviens très bien, où on travaillait avec des jeunes de Vénissieux. On testait nos premières pièces, qui confrontaient un spectateur avec des visions. J'ai été frappé par le fait que ces jeunes étaient toujours en groupe, et qu'on allait s'adresser à eux individuellement. Il fallait qu'ils acceptent d'assister seuls à cela. Je me suis donc vu construire ce sas de décompression pour eux, au moment de leur accueil.

JM : Notre travail ne fonctionne que si le spectateur s'implique. S'il ne s'implique pas, s'il ne veut pas se laisser aller et rester dans un rapport distant et consumériste, il n'y aura rien à comprendre, rien à tirer du travail, même pas une jolie histoire. Il aura juste vu de belles lumières. En franchissant la porte de l'appartement qu'on avait créé, ces jeunes de Vénissieux entraient chez nous, ils ne maîtrisaient pas toutes les règles. Il fallait qu'ils prennent conscience de cela, et qu'ils n'entrent pas en résistance.

AP : Faire entrer le spectateur dans votre monde, et faire en sorte qu'il n'ait pas l'impression d'être agressé, cela déplace le lieu de l'entrée en scène. Comment vous posez-vous la question du dedans et du dehors dans vos dispositifs ?

JM : Lors d'un entretien que j'ai fait au TU de Nantes ${ }^{4}$, il y avait une dame qui s'en voulait et qui était en colère car elle n'avait pas réussi à profiter du spectacle (Des Anges mineurs). Le monde qu'elle s'était construit ne lui permettait plus d'aller voir des spectacles qui duraient quatre heures et d'entrer dans ces mondes-là. Il y a eu un petit débat avec Loïc Touzet sur cette question : soit tu considères que tu amènes ton univers aux gens, qu'ils ouvrent la porte du théâtre et qu'ils entrent dedans sans problème et que c'est normal. Soit tu es conscient du fait que ça fait six mois que toi tu te prépares à entrer dans cet univers et à vivre dedans, mais que ce n'est pas du tout le cas du spectateur, qui, quand il franchit la porte, a autre chose dans la tête et n'est pas du tout préparé. Il vient d'ailleurs. D'où la nécessité de ce sas de pression ou décompression selon les narrations ou les univers. C'est comme dans les spectacles des STAN. Leur présence au plateau quand on rentre dans la salle, c'est une étape de décompression et de familiarisation avec eux, avec leur corps, leur présence, avec qui ils sont. Cela nous oriente sur ce dans quoi on va rentrer. C'est pareil pour chez nous, il faut accompagner cette descente. Dans Urbik/Orbik, c'est cette longue introduction, dans Des Anges mineurs, ce sont les mannequins de grand-mères qui apparaissent au milieu des spectateurs et qui leur permettent d'échanger autour de ça. Dans le prochain projet, on fera déborder l'espace de la représentation dans la salle. Les gens rentreront directement dans un espace scénographié, comme dans Le Bardo.

AP : Cette image de sas de décompression est souvent utilisée par les comédiens quand ils décrivent l'entrée en scène. Pour vous, il est vraiment du côté du spectateur.

$\mathrm{JM}$ : C'est d'autant plus flagrant dans Le Bardo d'ailleurs où quand on a construit les chambres, on a cherché à alterner les séquences qui mettaient davantage le spectateur en recul, dans un rapport contemplatif, avec des séquences qui le mettaient en prise plus directe. Ces temps de respirations, aménagés pour le 
spectateur, n'existent par contre pas vraiment pour l'acteur, qui joue en boucle pendant six heures, et ne fait qu'attendre l'entrée en jeu de son partenaire, le spectateur.

Le Bardo, La Chartreuse des Avignon, 7-14 juillet 2010

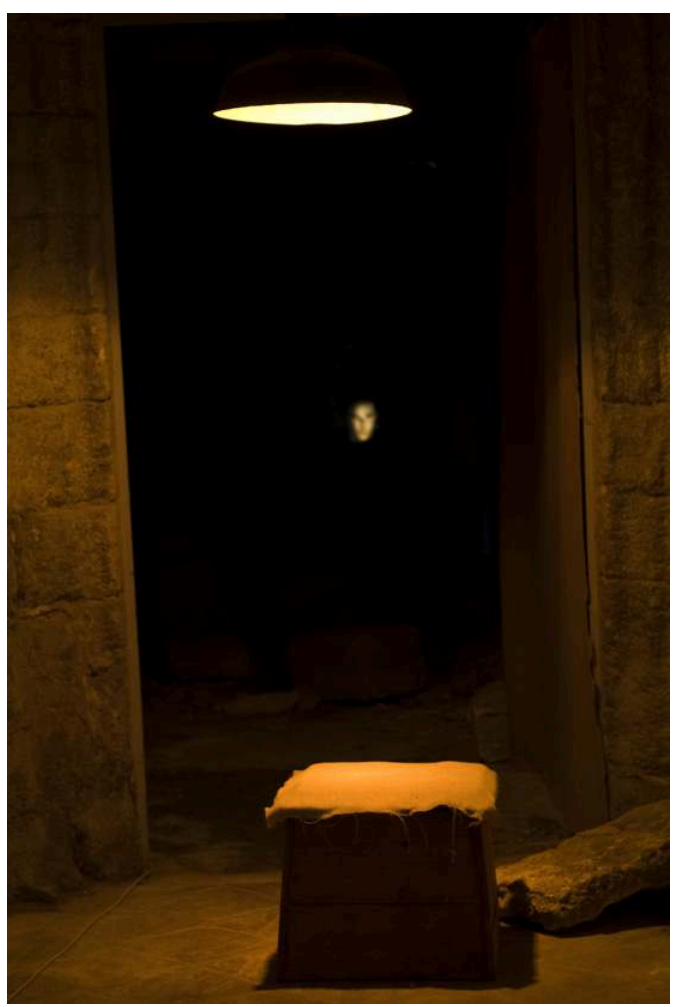

Nicolas Boudier

\section{Entrer nulle part pour ne rejoindre personne}

AP : Dans Urbik/Orbik, avec cette histoire des micro-mondes dans lesquels les personnages tombent, on a la sensation que vous voulez construire un espace utopique sur scène. Comment fait-on pour entrer nulle part?

PC : En croyant avec force qu'on entre quelque part. En tant qu'acteur, l'idée c'est de tellement croire à ce qu'on joue que les spectateurs vont être obligés de nous suivre dans cette histoire. Ça se passe à l'intérieur de l'acteur.

JM : Ta question me fait penser à une question qu'on nous avait posée dans une émission de radio. Le journaliste avait demandé à Philippe ce que ça lui faisait de disparaître sur scène tous les soirs. C'est une question juste, mais qui ne correspond pas à une réalité que l'on vit. Philippe n'a jamais l'impression de disparaître. L'enjeu est que les gens croient qu'il disparaît, qu'ils aient l'impression qu'un nulle part est en train de se créer. C'est ce noir dont tu parlais, aspirant, qu'on essaye de créer. Nous on n'entre jamais dans ce rapport de dupe. On continue à croire dur comme fer à notre table rouge et à notre ficelle. Les acteurs doivent croire aux situations qu'ils jouent. Philippe doit avoir l'impression qu'il parle à sa femme quand sa femme entre, même si c'est sous forme d'hologramme. Ce qui crée cette sensation, ce sont ces présences qui se disloquent plus qu'elles ne sortent de scène, ou qui apparaissent plus 
qu'elles n'entrent. C'est tout ce mélange-là qui fabrique ce nulle part, mais il n'y a pas de nulle part. Le lieu est bien ancré et les acteurs le sentent en permanence. Sur Urbik/Orbik par exemple, le plateau est en pente, il faut descendre des échelles... La conscience d'être présent sur la scène est permanente.

AP : Qu'est-ce que ça fait d'entrer en jeu avec pour partenaire quelqu'un qui n'existe pas, que ce soit une voix ou une image optique ou scénique?

PC : C'est une habitude de travail chez nous, qui est assez aisée à assimiler pour moi, mais qui a été plus difficile pour Marc ou Odile qui, sortant d'école, ont appris autre chose. Ils ont dû désapprendre. Pour ce qui est des cas spécifiques comme Le Bardo où le partenaire de jeu est le spectateur, c'est encore différent, c'est une excitation. Le risque de l'accident existe. Dans Le Bardo, je vois un spectateur qui est non pas en face de moi mais en face de mon fantôme. Ce fantôme est apparu en lieu et place d'une présence réelle, une jeune femme. Quand j'appuie sur un bouton, mon image apparaît et je vois le spectateur réagir à cette image fantomatique, le changement s'opérer sur son visage, et son trouble. Je l'appelle au téléphone, il doit me répondre et je l'oriente sur une conversation. Il suffit qu'il ne décroche pas pour que la machine se grippe, ou bien qu'il décroche mais n'ait pas envie de jouer le jeu. La question devient alors: comment faire pour que ce qui devait avoir lieu ait lieu?

JM : L'intérêt de ce type de séquence, c'est que ta fonction doit être rassurante, même si elle ne fait qu'amplifier l'angoisse. Dans le rapport d'interactivité que décrit Philippe, on laisse finalement peu de place au dialogue. Notre structure n'est pas chamboulée par la parole des spectateurs, parce que cela ne nous semble pas pertinent. Quand le spectateur prend la parole et parle trop, il reprend le contrôle. Il se rassure, recrée son propre univers et quitte celui dans lequel on veut l'amener.

$\mathrm{PC}$ : Pour revenir à Urbik/Orbik, et notamment à une scène où je dialogue avec mon psy qui est une voix enregistrée, la voix est ici un vrai partenaire de jeu. C'est très excitant à faire car on risque là aussi l'accident, le chevauchement des voix. Le partenaire de jeu virtuel est palpable. Ce n'est pas comme la vidéo qu'on ne voit pas. On entend la voix et on peut vraiment jouer avec. C'est une séquence que j'aime beaucoup.

JM : Ça change fondamentalement le rapport du comédien au plateau et à ses partenaires de jeu. Même dans l'évolution de notre travail, il y a des choses dont on sait qu'on se prive: des qualités qu'il y a dans un jeu plus direct, de dialogue, de présence, dans un rapport théâtral plus brut. Ce sont des choses qui peuvent nous manquer. La physicalité du jeu, la puissance, l'excitation au plateau. C'est parfois pesant, les comédiens le disent. Il ne faut pas que ces dispositifs deviennent une norme de jeu.

PC : Sur les deux derniers spectacles, on ne fait que se croiser. Il y a peu de séquences où l'on est en jeu ensemble. Parfois l'on sait qu'on est en jeu juste pour faire de l'image. Mais ça n'est pas désagréable, on n'a pas besoin de parler aux autres, on est tranquilles!

AP: J'ai l'impression que votre dispositif travaille intensément sur la singularité de la présence scénique et sur son ambiguiité : ce qu'il y a sur scène est à la fois ce qu'il y a sur scène et en même temps tout autre chose, justement parce que c'est sur scène. Ou 
encore : la présence de l'acteur en scène est hybride : il est à la fois lui-même et en même temps le personnage.

$\mathrm{JM}$ : Ça répond tout à fait à une interrogation qui traverse le théâtre depuis ses origines. Cet espace de la métaphore, de la projection du réel mais différé, la réflexion sur l'acteur, son corps et sa véracité sur scène. Moi, j'ai envie de raconter des histoires de science-fiction, de morts vivants. Et pourtant je n'ai envie de voir, ou de n'apprécier que du théâtre qui est extrêmement réel. J'ai besoin qu'on croie ce qui se passe sur scène, de manière hyper-réaliste. Et en même temps tu as raison, je veux que ce soit toujours hybride, que ce statut singulier de l'acteur et de l'illusion soit souligné. Il y a une phrase de Hesse que j'aime beaucoup dans Le Loup des Steppes : il rencontre un type dans la rue et voit une inscription néon qui l'attire, sur laquelle il croit lire : «Théâtre magique. Tout le monde n'entre pas. Seulement pour les fous ». J'aime bien cette idée là du théâtre qui accepte que tout le monde n'entrera pas, que ce soit l'endroit où la folie soit une qualité, l'endroit où l'on accepte de quitter la norme. Je trouve cette citation très juste, dans le rapport avec notre travail.

\section{NOTES}

1. Marc Menahem et Odile Ernoult. Promotion W. Sortis de l'école en juin 2011.

2. Des Anges mineurs, d'après le roman du même nom d'Antoine Volodine. Création le 4 décembre 2007 à la comédie de Saint Étienne.

3. Le Bardo, d'après Bardo or not Bardo d'Antoine Volodine. Mise en scène Joris Mathieu. Création le 12 janvier 2010 au Théâtre de Vénissieux.

4. Cet entretien a eu lieu lors d'une journée d'étude scientifique et artistique sur le thème suivant : «L'Utopie et ses représentations », organisé par le TU (Théâtre Universitaire) de Nantes et l'UFR de lettres et langages de l'Université de Nantes, le 25 mars 2011.

\section{INDEX}

Mots-clés : dispositif, jeu, mise en scène, théâtre optique 